\title{
Size-dependent deformation behavior of dual-phase, nanostructured CrCoNi medium-entropy alloy
}

\author{
Yujie Chen ${ }^{1,2,3}$, Xianghai $\mathrm{An}^{3^{*}}$, Zhifeng Zhou ${ }^{4}$, Paul Munroe ${ }^{5}$, Sam Zhang ${ }^{1^{*}}$, Xiaozhou Liao ${ }^{3}$ and \\ Zonghan $\mathrm{Xie}^{2,6}$
}

\begin{abstract}
The mechanical size effect of nanostructured, dual-phase CrCoNi medium-entropy alloy (MEA) was investigated by combining in-situ micro-compression testing with post-mortem electron microscopy analysis. The alloy possesses a superior yield strength up to $\sim 4 \mathrm{GPa}$, primarily due to its hierarchical microstructure including column nanograins, preferred orientation, a high density of planar defects and the presence of the hexagonal close packed (HCP) phase. While the yield strength of the alloy has shown sizeindependency, the deformation behaviour was strongly dependent on the sample size. Specifically, with decreasing the pillar diameters, the dominant deformation mode changed from highly localized and catastrophic shear banding to apparently homogeneous deformation with appreciable plasticity. This transition is believed to be governed by the sizedependent critical stress required for a shear band traversing the pillar and mediated by the competition between shearinduced softening and subsequent hardening mechanisms. In addition, an unexpected phase transformation from HCP to face-centered cubic (FCC) was observed in the highly localized deformation zones, leading to strain softening that contributed to accommodating plasticity. These findings provide insights into the criticality of sample dimensions in influencing mechanical behaviors of nanostructured metallic materials used for nanoelectromechanical systems.
\end{abstract}

Keywords: medium-entropy alloy, size effect, shear banding, phase transformation, nanostructure

\section{INTRODUCTION}

High- and medium-entropy alloys (HEAs and MEAs), often referred to as multi-component alloys, have emerged as a new class of metallic materials with excellent physical and mechanical properties over the last decade [1-4]. The original design concept of these novel alloys was proposed to maximize the configurational entropy for the formation of single-phase solid solution by mixing multiple alloying elements having near equiatomic concentrations $[5,6]$. It is increasingly realized that the Gibbs free energy in lieu of this type of entropy plays predominant roles in the phase formation and its stability, enabling that the original strict restriction for the development of HEA/MEAs is thermodynamically relaxed [79]. This can tremendously increase compositional space for this kind of novel alloys and catalyze novel mechanism-driven HEAs/MEAs design. For instance, twinninginduced plasticity (TWIP) and transformation-induced plasticity (TRIP) effects can be introduced into these alloys through the optimization of their stacking fault energy (SFE) via tuning their chemical compositions and concentrations, leading to the improved mechanical properties [10-13]. It is well acknowledged that the CrCoNi MEA with face-centered cubic (FCC) structure exhibits an unprecedented combination of strength, ductility, and toughness, as compared with the prototype of the CrCoNiFeMn HEA [14]. Extensive investigation proposed that these extraordinary mechanical properties might be attributed to its lower SFE, enabling the extensive twinning activities and the development of hexagonal close packed (HCP) nanolaths in CrCoNi via an FCC to HCP phase transformation [15-17].

More recently, the design principles are dedicated to

\footnotetext{
${ }^{1}$ Centre for Advanced Thin Films and Devices, School of Materials and Energy, Southwest University, Chongqing 400715, China

${ }^{2}$ School of Mechanical Engineering, The University of Adelaide, Adelaide, SA 5005, Australia

${ }^{3}$ School of Aerospace, Mechanical and Mechatronic Engineering, The University of Sydney, Sydney, NSW 2006, Australia

${ }^{4}$ Department of Mechanical Engineering, City University of Hong Kong, Kowloon, Hong Kong, China

${ }^{5}$ School of Materials Science and Engineering, The University of New South Wales, Sydney, NSW 2052, Australia

${ }^{6}$ School of Engineering, Edith Cowan University, Perth, WA 6027, Australia

* Corresponding authors (emails: xianghai.an@sydney.edu.au (An X); samzhang@swu.edu.cn (Zhang S))
} 
incorporating other strengthening mechanisms into this kind of novel alloys, such as microstructure modification [18-20], precipitation strengthening [21,22], ductile multicomponent intermetallic nanoparticles [23], and interstitial solid solution strengthening (by adding boron [24], carbon [25], nitrogen [26,27] and oxygen [28]), to push the property boundary of possibility apart from their inherent substitutional solid solution strengthening. Similarly, we successfully fabricated a nanostructured dual-phase (HCP and FCC) CrCoNi MEA using magnetron sputtering, which possesses ultrahigh nanohardness of $\sim 10 \mathrm{GPa}$ obtained by nanoindentation that is much higher than that of conventional FCC structured counterpart $[29,30]$. It was revealed that this ultrahigh hardness originated from its unique hierarchical microstructures enabled by the special processes of magnetron sputtering [29,30]. However, the detailed mechanical behaviors of the newly fabricated dual-phase CrCoNi MEA in terms of stress-strain curves, deformability and underlying deformation mechanism have not been systematically explored and especially, the ductility achievable under such high hardness remains mysterious, both of which will be essentially uncovered in the current investigation.

As well known, the sample size can be a crucial factor in regulating the mechanical behaviour of structural materials when the sample size is reduced to the micron scale and below [31-33]. Notable observations include the inversely proportional relationship between strength and sample sizes, i.e., "smaller is stronger", which is generally associated with appreciable reduction in structural defects and dislocation starvation [32,33] caused by the inability of sub-micron or nanoscale samples to store dislocations. In addition, the deformation mode of single crystal pillars is also size-dependent. For example, the deformation in $\mathrm{Al}$ and $\mathrm{Ni}$ single crystal pillars was dominated by homogeneous plastic flow at the micron scale, while large strain bursts and dramatic collapse took place in submicron pillars as a result of dislocation avalanche $[31,34]$. However, when the complex microstructures featuring grain boundaries (GBs), planar defects and precipitates are incorporated in the materials, the presence of these structural defects in small-scale specimens may preclude known mechanisms responsible for the size-dependent plasticity of materials [31,32]. For instance, the size effect on the mechanical behavior of alloys with a high density of growth planar defects remains largely unexplored. In light of the high performance of the MEAs and their massive potential in the application of nano- or microelectromechanical systems (NEMS/MEMS) [35], it is imperative to understand the mechanical response of MEAs containing complex microstructures as a function of sample size, which will not only enrich our understanding of size-related deformation of novel materials, but also provide valuable guideline to the design and development of reliable, high-performance NEMS/MEMS [35].

In this study, a nanostructured, dual-phase $\mathrm{CrCoNi}$ alloy containing a large number of growth planar defects was prepared using magnetron sputtering. In-situ compression testing of micro-pillars with diameters ranging from 2 to $0.23 \mu \mathrm{m}$ was performed. The size effect on the mechanical behaviors of the alloy was systematically explored. Compared with conventional FCC structured CrCoNi, this newly created alloy showed a superior yield strength of $\sim 4 \mathrm{GPa}$. While the yield strength of the alloy was found to be essentially size-independent, the deformation mode was strongly dependent on the sample size. As the diameter decreases, a transition in the deformation mode occurred in the alloy from brittle failure, caused by unsteady large shear banding, to 'ductile' deformation (i.e., uniform deformation) accompanied by minor shear events. Moreover, an unexpected HCP to FCC phase transformation was identified, indicating the HCP phase is unstable under applied stress.

\section{EXPERIMENTAL SECTION}

The CrCoNi alloy was deposited on AISI M2 steel substrates (hardened to HRC 62, and polished to a final surface roughness of $0.01 \mu \mathrm{m}$ ) using a direct current (DC) magnetron sputtering system. A CrCoNi target (Cr:Co: $\mathrm{Ni}=1: 1: 1$, at $\%$ ) with the purity of $>99.9 \%$ and dimensions of $345 \mathrm{~mm} \times 145 \mathrm{~mm} \times 5 \mathrm{~mm}$ was used for the alloy deposition. The substrates were first ultrasonically cleaned before mounting on a stationary holder. The target-tosubstrate distance was set at $170 \mathrm{~mm}$. The background chamber was pumped down to a pressure of $4 \times 10^{-4} \mathrm{~Pa}$. Prior to deposition, the substrates were ion cleaned for $30 \mathrm{~min}$ under a bias voltage of $-450 \mathrm{~V}$ to remove any contaminants on the substrate surfaces. The argon (Ar) working gas (99.995\% purity) was fed into the chamber at a constant flow rate of $50 \mathrm{sccm}$ by a MKS mass flow controller and the chamber pressure was kept at $0.17 \mathrm{~Pa}$ during sputtering. No external heating was used and a bias voltage of $-60 \mathrm{~V}$ was maintained during the coating deposition. The CrCoNi DC was maintained at $4.0 \mathrm{~A}$ (sputtering power of $\sim 1.5 \mathrm{~kW}$ ), corresponding to a nominal deposition rate of $\sim 72 \mathrm{~nm} \mathrm{~min}^{-1}$. The $\mathrm{CrCoNi}$ film was deposited with a nominal thickness of $5 \mu \mathrm{m}$ 
directly onto the steel substrates.

Micro-pillars for uniaxial micro-compression tests were fabricated using a dual beam scanning electron microscope/focused ion beam (SEM/FIB) system (FEI Helios Nanolab 600) [36]. Three steps were involved; namely, coarse milling, fine milling and a final superfine polishing. The beam current was reduced to $9.7 \mathrm{pA}$ at the final polishing step to minimize FIB damage. The FIB-milled pillars had varying diameters $(d)$ ranging from $\sim 230 \mathrm{~nm}$ to $\sim 2 \mu \mathrm{m}$, and aspect ratios of the height $(h)$ to $d$, ranging from $\sim 2.5$ to $\sim 3$. The tapering angle of the pillars ranged from $\sim 2^{\circ}$ to $\sim 4^{\circ}$. Diameters measured at the top of the pillars were used to calculate the engineering stress, since for most of the pillars the deformation was mainly localized in the upper region [37,38]. The compression tests were performed in an SEM equipped with a nanoindenter (PI 85 SEM PicoIndenter, Hysitron Inc.) containing a $5-\mu \mathrm{m}$ diameter diamond flat punch. The compression tests were performed in the displacement control mode at a prescribed strain rate of $10^{-3} \mathrm{~s}^{-1}$. The pillars were deformed to a prescribed displacement (or strain) followed by an incremental unloading.

The overall morphology of the post-compressed pillars was characterized by a dual beam SEM/FIB at $10 \mathrm{kV}$. Thin-foil transmission electron microscopy (TEM) samples of both the as-deposited films and the pillars after compression were prepared by using an in-situ lift-out technique [39] inside the same dual beam SEM/FIB system. The TEM lamellae were finally polished with a $5 \mathrm{kV}$ and $24 \mathrm{pA} \mathrm{Ga}{ }^{+}$ion beam to minimize ion beam damage to the samples. Bright-field diffraction contrast imaging and selected area diffraction (SAED) were performed by using a Philips CM200 TEM operating at $200 \mathrm{kV}$. Highangle annular dark-field (HAADF) scanning TEM (STEM) observations and compositional mapping were conducted by using an aberration-corrected FEI Titan Themis TEM operating at $200 \mathrm{kV}$.

\section{RESULTS}

\section{Hierarchical nanostructures of the dual-phase $\mathrm{CrCoNi}$ MEA}

As illustrated in Fig. 1, the as-deposited CrCoNi alloy exhibits a hierarchical nanostructure. The characteristic microstructural feature of the alloy is the columnar grains with widths ranging from $\sim 50$ to $150 \mathrm{~nm}$ (Fig. 1a). At the atomic scale, typical STEM images of two columnar 'grains' (Fig. 1b, c) reveal alternating HCP and FCC phases, as well as the presence of a high density of planar defects, including stacking faults (SFs) and twin boundaries (TBs). The FCC phase has a consistent orientation relationship with the HCP phase, specifically, $\{111\}_{\mathrm{F}} \|(0001)_{\mathrm{H}}$ and $\langle 110\rangle_{\mathrm{F}} \|\langle 1120\rangle_{\mathrm{H}}$. The grain texture was identified with the $[0001]_{\mathrm{H}}$ and $\langle\overline{1} 11\rangle_{\mathrm{F}}$ direction oriented along the film growth direction. Extensive STEM investigations further confirmed the presence of HCP and FCC segments, each having a thickness of $\sim 2$ to $8 \mathrm{~nm}$ and alternatively distributed through the columnar grains. The volume percentages of HCP and FCC phases were statistically estimated to be $\sim 60 \%$ and $\sim 40 \%$, respectively, based on the STEM images of 15 columnar grains. In principle, the hierarchical nanostructures of the asdeposited MEA with dual phases are significantly distinct from those of the coarse-grained counterpart with a single FCC phase structure fabricated by conventional metallurgical methods [14,17], endowing it with ultrahigh hardness and unique deformation behavior [29].

\section{Size-dependent deformation of the dual-phase $\mathrm{CrCoNi}$ MEA}

To comprehensively explore the mechanical behavior of the CrCoNi alloy, in-situ compression tests of pillars were conducted using samples with diameters ranging from 2 to $0.23 \mu \mathrm{m}$. It is apparent that the sample sizes have re-
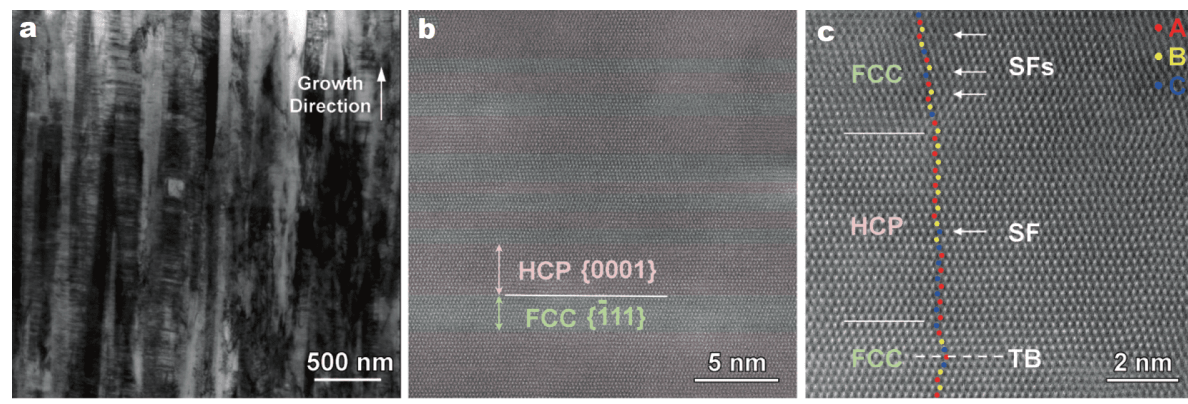

Figure 1 (a) A bright field TEM image of the as-deposited CrCoNi alloy showing the columnar structure containing a high density of planar defects; (b, c) STEM images of two separate grains showing the coexistence of HCP and FCC phases, as well as SFs and TBs. 
markable effects on the deformation behavior of the alloy, as revealed in Fig. 2. When the sample diameters were larger than $1 \mu \mathrm{m}$, pillars failed abruptly through major shear banding. In striking contrast, pillars with diameters below $1 \mu \mathrm{m}$ exhibited appreciable ductility controlled by steady shear banding. Fig. 2a, b show the undeformed morphology of a pillar with a diameter of $1.45 \mu \mathrm{m}$ (pillar 1) and its compression failure mode. The pillar initially showed elastic deformation, followed by limited strain hardening with the yield stress reaching $\sim 4.2 \mathrm{GPa}$ before the stress drop (Fig. 2c). The sudden drop in stress was caused by shearing of the top surface toward the righthand side of the pillar. Finally, the pillar failed catastrophically with a strain-to-failure of $\sim 5 \%$ through the formation of multiple major shear bands, indicated by white arrows, and the majority of the pillar body sheared toward the left-hand side. The compression test was per- formed under displacement-control, therefore extremely rapid propagation of these shear bands left the punch behind (moving at the preset displacement rate), resulting in a stress drop straight to zero.

In comparison, an as-fabricated pillar with a diameter of $0.88 \mu \mathrm{m}$ (pillar 2) and its post-compression morphology are presented in Fig. 2d, e, respectively. The corresponding engineering stress-strain curve is shown in Fig. 2f. Owing to the taper geometry of the FIB-fabricated pillar, the plastic deformation commenced from the pillar top surface that came into contact with the indenter. The top part of the pillar experienced a uniform plastic flow before the shear banding started. The stress decreased after point 1 due to the formation and development of the first shear band $\left(\mathrm{SB}_{1}\right)$. The stress decreased to a trough (point 2) when the $\mathrm{SB}_{1}$ ceased to propagate. Following that, the stress increased again until a second decrease
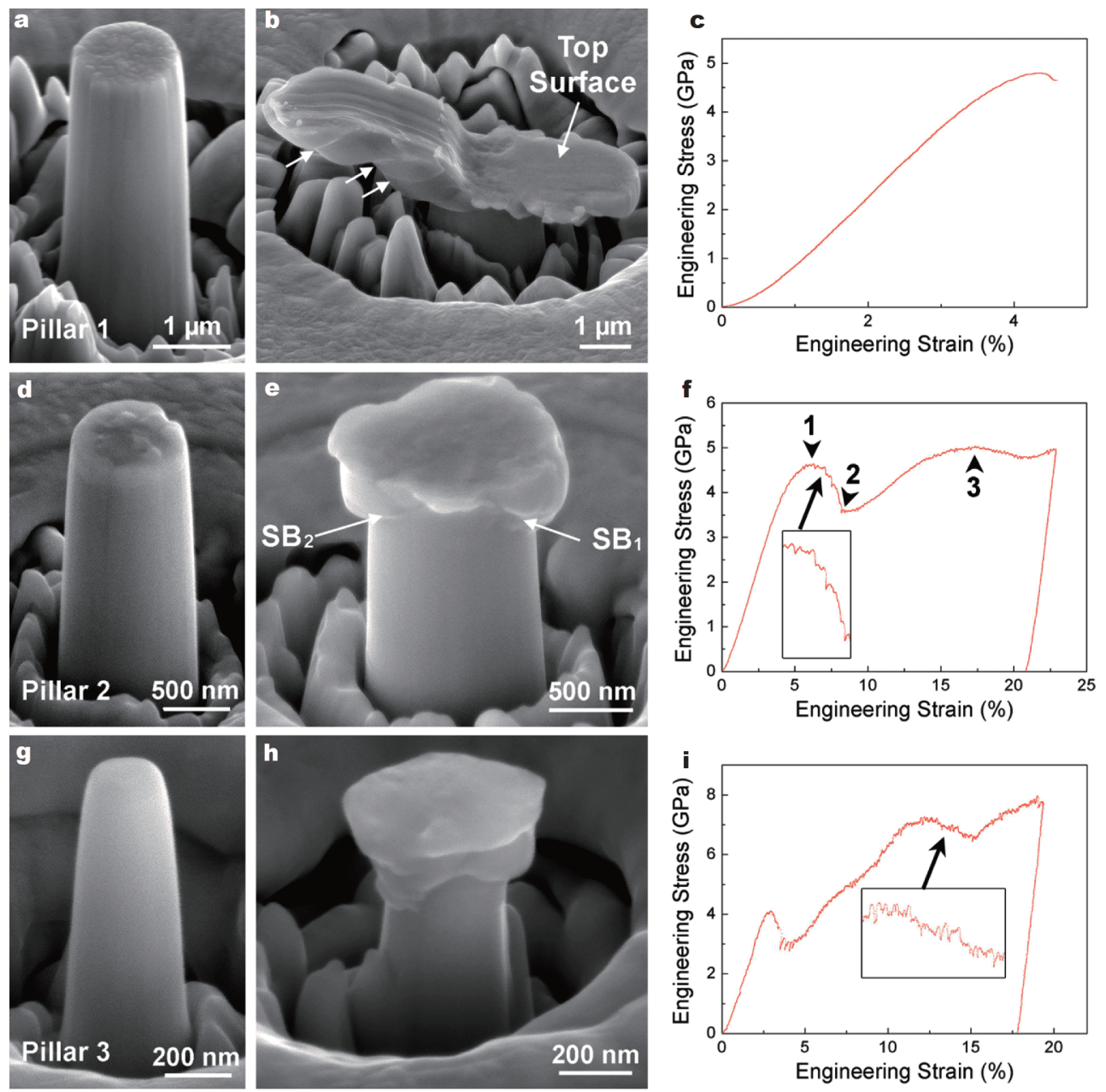

Figure 2 SEM images of undeformed and post-compressed pillars with diameters of (a, b) $1.45 \mu \mathrm{m},(\mathrm{d}, \mathrm{e}) 0.88 \mu \mathrm{m}$, (g, h) $0.23 \mu \mathrm{m}$; (c, f, i) engineering stress-strain curves corresponding to the pillars in $(a, d, g)$, respectively. 
occurred at point 3 caused by the initiation of the second shear band, as indicated by $\mathrm{SB}_{2}$ (Fig. 2e). The indenter retracted automatically when it reached the prescribed maximum displacement. Different from the catastrophic shear failure of pillar 1, the propagation of the shear bands in Fig. 2e was relatively slow and no large displacement bursts was observed. As shown in the inset in Fig. 2f, the curve does not exhibit a smooth stress response. The intermittent stress drops were presumably due to successive controlled steady shears in the same band.

With further reductions in the pillar diameter, uniform and steady plastic flow became dominant during compression testing. Fig. 2g, h display the morphology of a pillar with a diameter of $0.23 \mu \mathrm{m}$ (pillar 3) before and post deformation. The corresponding engineering stressstrain curve is shown in Fig. 2i. Plastic deformation was mainly confined at the top of the slightly tapered pillar, which changed the pillar shape to a "mushroom-like" morphology, indicative of considerable plasticity in the pillar top. The stress response after a strain of $5 \%$ presents "apparent hardening" in the engineering stress-strain curve, which may result from the increase of the contact area between the indenter and the pillar tip with continued compression. The pillar shows 'ductile' deformation with some intermittent stress drops apparent in Fig. 2i.

The yield strength was measured and plotted in Fig. 3

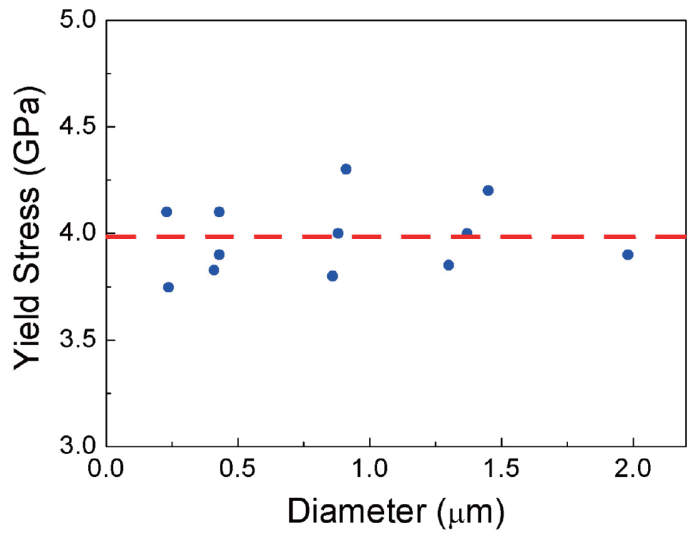

Figure 3 Yield stress versus pillar diameter for nanostructured $\mathrm{CrCoNi}$ alloy pillars.

as a function of pillar diameter. Different from the "smaller is stronger" phenomenon now well-known for most crystalline materials [31,40], it turns out that for the hierarchically nanostructured $\mathrm{CrCoNi}$ alloy there is no apparent dependence of yield strength on the pillar diameter over the size range covered in this work.

\section{Deformation mechanisms of the dual-phase CrCoNi MEA}

To obtain a better understanding of the deformation mechanisms at the atomic level, the microstructure of the regions both within and around the shear bands of the post-compressed pillars was analyzed by using STEM
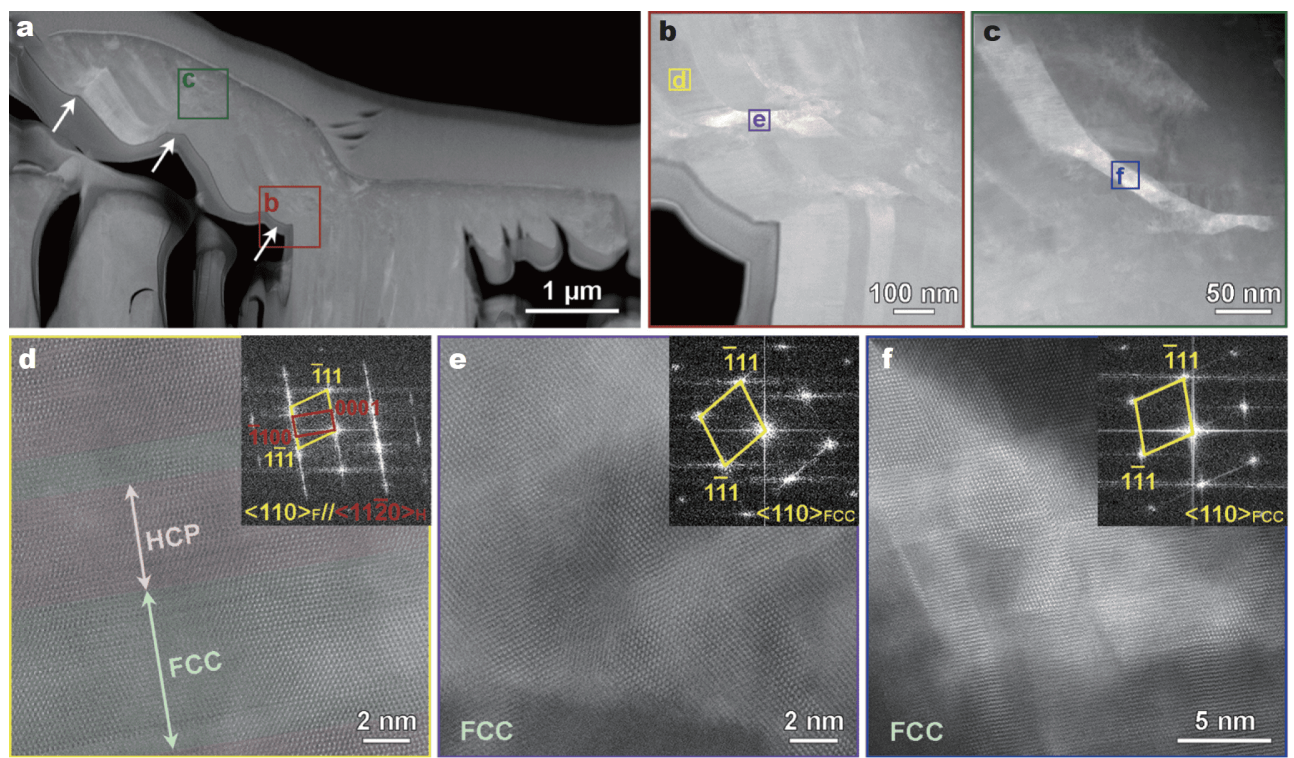

Figure 4 (a) A STEM image of the post-compressed pillar with a diameter of $1.45 \mu \mathrm{m}$ failed catastrophically via shear banding. STEM images of the regions enclosed by (b) red rectangle, and (c) green rectangle in (a) showing the structure of shear bands. Magnified STEM images and the corresponding FFT (inset) of three selected regions: (d) near the shear band and (e) inside the shear band shown in (b), and (f) inside the shear band shown in (c). 
imaging. Fig. 4 exhibits the postmortem microstructures of the pillar presented in Fig. $2 \mathrm{~b}(1.45 \mu \mathrm{m}$ diameter) that failed catastrophically during the compression testing. Three shear bands, as indicated by white arrows, are detected in the part of the pillar that sheared to the left. The shear band regions enclosed by red and green rectangles are magnified and shown in Fig. 4b, c, respectively. The grains inside the shear bands still possess a columnar structure but are sheared to the left, forming an angle with respect to the less deformed columnar grains. In the region next to a shear band enclosed by the red rectangle in Fig. 4b, similar to those in the as-deposited film, the HCP and FCC structures still coexist as revealed in the magnified STEM micrograph presented in Fig. $4 \mathrm{~d}$ and its corresponding fast Fourier transform (FFT) pattern (inset). However, only the FCC phase was observed within the shear band, as evidenced in the STEM image in Fig. $4 \mathrm{e}$ taken from the same grain as Fig. 4d, f obtained from another shear band presented in Fig. $4 \mathrm{c}$, as well as their corresponding FFT patterns (inset). These findings signal that plasticity localized within the shear bands and that the phase transformation from HCP to FCC unexpectedly occurred during shear banding.

Fig. 5a presents an STEM image of pillar $2(0.88 \mu \mathrm{m}$ diameter) shown in Fig. 2e, indicating the pillar was de- formed mainly by two shear bands $\left(\mathrm{SB}_{1}\right.$ and $\mathrm{SB}_{2}$ marked by white dash lines). The slight loss of the top-right corner of the pillar was due to FIB milling. A magnified image presented in Fig. $5 \mathrm{~b}$ demonstrates the microstructures within the $\mathrm{SB}_{2}$ (the bright contrast region) with a thickness of $\sim 40 \mathrm{~nm}$ enclosed by a red rectangle shown in Fig. 5a. The atomic structure within the shear band enclosed by a green rectangle in Fig. $5 \mathrm{a}$ and the corresponding FFT pattern (inset), as exhibited in Fig. 5c, validate the existence of a single FCC structure in lieu of the FCC and HCP phases, which is in line with those in Fig. $4 \mathrm{e}, \mathrm{f}$. In addition to the phase transformation from HCP structure to FCC structure, other dislocation activities were also frequently detected within the shear bands. As indicated by the high-resolution inverse FFT (IFFT) images (Fig. 5d, e), Lomer-Cottrell (L-C) locks formed in the shear bands. In general, when two extended dislocations on two intercrossing $\{111\}$ slip planes meet each other, the two leading partials form a stair-rod dislocation by the following reaction:

$\frac{1}{6}[\overline{1} \overline{2} \overline{1}]+\frac{1}{6}[211] \rightarrow \frac{1}{6}[1 \overline{1} 0]$.

The stair-rod dislocation and the two trailing partial dislocations form a sessile L-C lock dislocation with a Burgers vector of $\frac{1}{2}[1 \overline{1} 0]$. Extra planes can be seen for
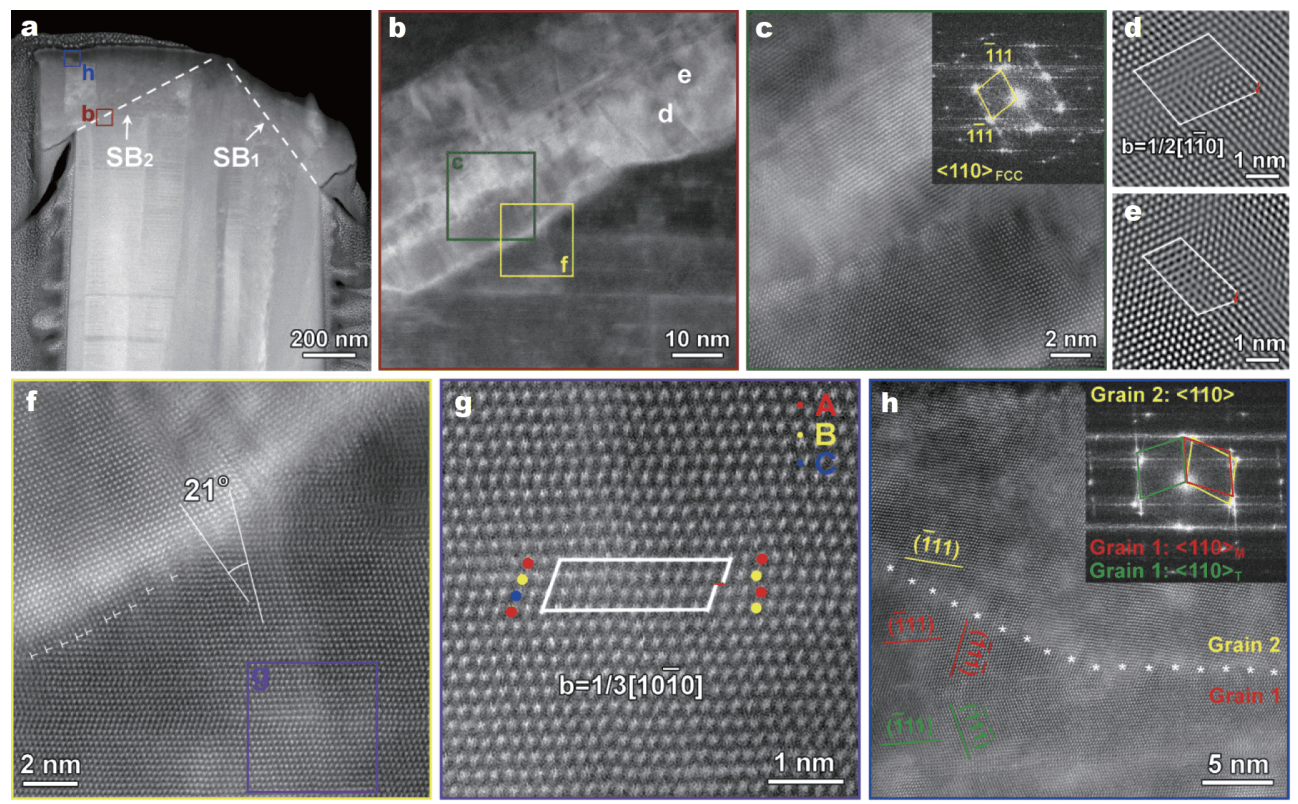

Figure 5 (a) An STEM image of the deformed pillar with a diameter of $0.88 \mu \mathrm{m}$ in Fig. $2 \mathrm{~d}$, showing the two shear bands $\left(\mathrm{SB}_{1}\right.$ and $\left.\mathrm{SB}_{2}\right)$. (b) $\mathrm{A}$ magnified STEM image of part of $\mathrm{SB}_{2}$. (c) An STEM image and its corresponding FFT (inset) exhibiting the FCC structure inside the shear band. (d, e) IFFT images of an L-C lock dislocation configurations inside the shear band. (f) An STEM image of the boundary between the sheared and un-sheared region indicating the misorientation and dislocations at the shear band boundary. $(\mathrm{g}$ ) A shockley partial dislocation at FCC/HCP interfacial region. (h) An STEM image and its corresponding FFT (inset) demonstrating the highly deformed region beneath the top surface of the pillar that exhibits FCCphased nanograins with the twin structure. 
both the (111) and the (111) planes. It has been reported that L-C locks play a critical role in the strain hardening of FCC metals, because they are effective barriers to mobile dislocations [41]. Therefore, a higher stress is required during the later stages of shear band propagation. Since L-C locks formed via the reaction of leading partials of extended dislocations, their formation are substantially catalyzed in low SFE materials, such as the CrCoNi MEA [42].

The structure of the shear band boundary can be found in Fig. 5f. The regions inside (top) and right below (bottom) the shear band, exhibit a single FCC phase with a misorientation angle of $21^{\circ}$, indicating an anticlockwise crystallographic rotation inside the shear band. It is most likely that the anticlockwise lattice rotation is caused by the geometry of the shear deformation. A large shear strain is introduced to the rotated $\{111\}_{\mathrm{F}}$ or $(0001)_{\mathrm{H}}$ plane inside the shear band, since it is parallel to the shear direction, facilitating the dislocation activities on the $\{111\}_{\mathrm{F}}$ or $(0001)_{\mathrm{H}}$ plane. Further, the shear band boundary consists of high density of dislocations, as marked by the white $\perp$ signs, which mainly leads to the misorientation across the shear band. The STEM image of an FCC/HCP interfacial region (Fig. 5g), enclosed by a violet rectangle in Fig. 5f, can substantiate that the FCC phase is formed via the glide of Shockley partial dislocations with a Burgers vector of $\frac{1}{3}\langle 1010\rangle$. In addition to the shear bands, a highly deformed region enclosed by the blue rectangle beneath the top surface in Fig. 5a was also carefully characterized, as demonstrated by an STEM image and corresponding FFT pattern (inset) shown in Fig. 5h. FCC-structured nanograins with twins can be observed in this region. The twin thickness in the deformed regions is $\sim 10$ to $20 \mathrm{~nm}$, which is much thicker than those in the as-deposited samples, indicative of the possible occurrence of detwinning. Meticulous examination of the structure in the highly deformed regions from four pillars with different diameters found that the HCP phase rarely presented, verifying the occurrence of a phase transformation from the HCP to FCC phase in these areas, which was essentially distinct from the transformation from FCC to HCP that occurred in the coarse-grained counterparts $[17,43]$.

\section{DISCUSSION}

\section{Size-independent ultra-high strength}

The experiments described above show that the nanos- tructured CrCoNi pillars exhibit a size-independent yield strength of $\sim 4 \mathrm{GPa}$, which is a factor of $\sim 10$ (i.e., an order of magnitude) higher than that of bulk FCC $\mathrm{CrCoNi}$ MEA [14,17]. The hardness of the CrCoNi film, measured by nanoindentation, is $\sim 10 \mathrm{GPa}$ [29], about five times increase as compared with that of the as-cast $\mathrm{CrCoNi}$ $(\sim 1.86 \mathrm{GPa})$ [44]. The ultra-high yield strength and hardness are believed to arise from the hierarchical nanostructural features of the $\mathrm{CrCoNi}$ film, including column nanograins, a high density of planar defects and $\mathrm{HCP} / \mathrm{FCC}$ phase interfaces, strong $[0001]_{\mathrm{H}}$ and $\langle 111\rangle_{\mathrm{F}}$ directional texture, and a large fraction of the HCP phase. It is well known that the GB strengthening efficiency of CrCoNi-based HEAs and MEAs is significantly larger than conventional FCC alloys [45], which reasonably enables the high strength of nanostructured HEA and MEA. In addition, within individual column nanograins, the high density of planar defects including SFs, TBs and phase boundaries (PBs) plays crucial roles in strengthening the materials since they are effective in impeding the transmission of dislocations on the slip systems inclined to these defects [46-49].

Recent investigations revealed that the deformationinduced HCP phase in bulk HEA and MEA are stronger than their FCC counterparts [50]. Therefore, the presence of HCP phases in the nanostructured CrCoNi films unambiguously results in a higher yield strength than its single FCC structure. Moreover, the growth direction of the $\mathrm{CrCoNi}$ film is dominated by $[0001]_{\mathrm{H}}$ and directions (i.e., it exhibits a strong texture). The easy glide plane in the HCP phase is the (0001) basal plane, which is normal to the loading direction, thus resulting in a lack of resolved shear stress for dislocation motion on the basal plane. Further, the activation of $\langle\mathrm{c}\rangle$ and/or $\langle\mathrm{c}+\mathrm{a}\rangle$ dislocations on the pyramidal or prismatic slip planes can be inhibited by the high density of SFs. Similarly, the nucleation of dislocations is essentially suppressed in the FCC phase with profuse SFs and TBs due to their extremely small spacing [51,52]. Although dislocations can also glide along TBs that are orthogonal to the loading direction as well, there is no resolved shear stress for the migration of dislocation along TBs in the present study. The strong $[0001]_{\mathrm{H}}$ and $\langle\overline{1} 11\rangle_{\mathrm{F}}$ texture therefore increases the yield strength of both the HCP and the FCC phases. In principle, the ultrahigh strength of nanostructured CrCoNi MEA is endowed by the integrative effects stemming from hierarchical, heterogeneous microstructures across length scales from atomic level to nanometer, rather than by any specific microstructural 
features.

Although sample size effects on the strength of metallic materials have been widely acknowledged, there is no dependence of the yield strength on the pillar diameter observed over the size scale of the nanostructured CrCoNi alloy examined in this study. As mentioned above, the ultra-high yield strength in the CrCoNi pillars originates from the difficulty of dislocation motion due to the densely spaced planar defects. Actually, the wellknown "smaller is stronger" phenomenon observed in nano-sized crystalline materials originates from sophisticated dislocation activities [32,35]. Therefore, apparent size effects might not be expected here because dislocations are trapped inside the pillars by planar defects and phase boundaries and thus hardly move and escape from the free surface to reach a dislocation starvation state. Although size-induced weakening phenomena were also found in nanograined metal pillars due to the GB-assisted deformation [53], the special GB features endowed by the column grained structures can largely inhibit the GB migration and GB sliding. Therefore, the yield strength of current nanostructured CrCoNi MEA is essentially sizeindependent, which stems from its unique microstructures.

\section{Size-dependent deformability}

Unlike dislocation-mediated plastic flow observed in bulk CrCoNi, pillars made of a CrCoNi film in this study deform mainly through shear banding. Generally, when conventional modes of deformation with crystallographic features are inhibited, shear banding, as a form of local instability, will be activated to accommodate the plasticity, which is frequently observed in high-strength materials such as bulk metallic glasses and nanocrystalline materials [54-57]. It is also well known that localized deformation tends to occur when the ratio between the strain hardening rate and the applied stress drops below a critical value [58]. Therefore, the ultrahigh strength of the nanostructured $\mathrm{CrCoNi}$ film and its limited strain hardening at the early stage of deformation will essentially promote the localized deformation in the form of shear banding that appears at sites of stress concentration.

Although shear banding is the primary mechanism of plastic deformation, the remarkable sample size effects on the deformability of $\mathrm{CrCoNi}$ pillars are readily observed in the current study. With the diameters of the pillars gradually decrease from 2 to $0.23 \mu \mathrm{m}$, the deformation shows a transition from (1) highly localized and catastrophic shear banding to (2) slowly propagated and steady shear banding accompanied by softening, and eventually (3) "homogeneous-like" deformation with appreciable plasticity (i.e., a brittle to 'ductile' transition). The size effects on the plasticity of CrCoNi pillars do not follow the trend of that of single crystal pillars [31,34], but are similar to that of metallic glass pillars [54-56]. Actually, the size effects on the deformability of $\mathrm{CrCoNi}$ pillars can be understood by considering the critical stress required for shear band propagation to fracture $[59,60]$. While the initiation of shear banding is controlled by the shear stress, the propagation of the shear band is governed by the release of stored elastic energy [54]. For materials with a high density of obstacles, slip is confined in such a way that the elastic strain energy cannot be easily released by means of dislocation motion. The total elastic strain energy $U$ stored in a pillar with a diameter $d$ and a height $h$ due to the applied stress $\sigma$ can be obtained as [59]:

$U=\pi d^{2} h \sigma \varepsilon / 8$.

The consumption or release of the elastic energy, $\Delta U$, during the shear-banding process can be calculated by [59]:

$\Delta U=\sqrt{2} \pi d^{2} \Gamma / 4$,

where $\Gamma$ is the energy per unit area of shear band, which is assumed to be a constant, regardless of the sample size. Similar to what takes place during crack propagation (Griffith criterion) [61], the stored elastic energy $U$ would be completely relieved by the catastrophic shear band that traverses the pillar. Then, the size effect on deformation is rendered by the dimensional origin (i.e., three-dimensional energy stored in volume versus two-dimensional energy consumption by surface). Therefore, $\Delta U=U$ gives an estimate of the critical stress $\sigma_{\mathrm{c}}$ required for propagating a single shear band to fracture the pillar [59]:

$\sigma_{\mathrm{c}}=\sqrt{\frac{2 \sqrt{2} \Gamma \sigma / \varepsilon}{h}}=\sqrt{\frac{2 \sqrt{2} \Gamma E}{a d}}$,

where $E$ is Young's modulus, $a$ is the aspect ratio (height/ diameter). The critical stress required to raise the strain energy so that it was sufficient to enable the shear band propagation gradually to increase as the pillar diameter decreases (Fig. 6). Note that the yield stress is measured to be size-independent. Regarding the limited strain hardening in pillars with different diameters, it can be reasonably inferred that the flow stress that initiates shear band also exhibits size-independency. That means, there exists a critical diameter, $d_{c}$, where the transition from brittle to 'ductile' occurs (Fig. 6), since below this size, catastrophic shear banding becomes suppressed due to the insufficient elastic strain energy to fuel the expansion 


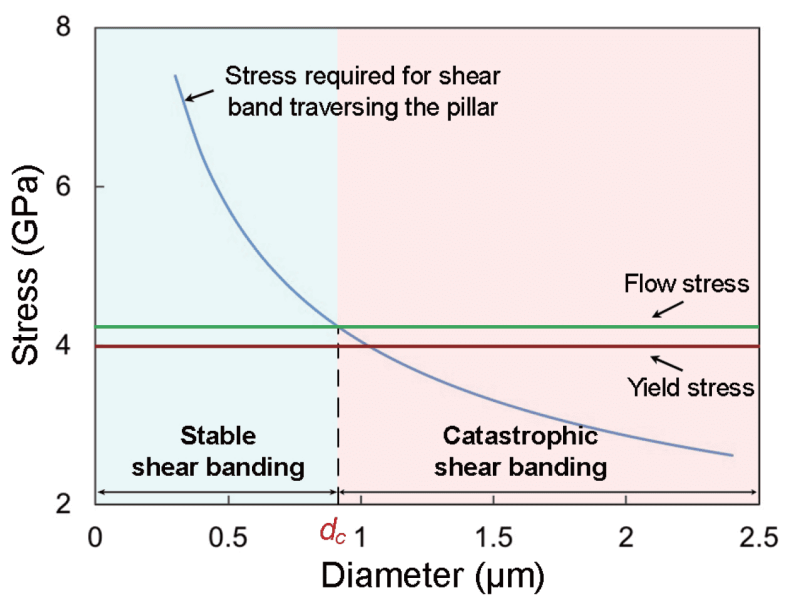

Figure 6 Schematic illustration of the transition from catastrophic unsteady shear banding to stable and slow shear banding with decreasing pillar diameter. The minimum stress required for a shear band to fracture the pillar (blue solid line) crosses the experimental-measured flow stress $(\sim 4.2 \mathrm{GPa})$ when shear band initiates (green solid line) at a critical diameter, $d_{c}$, where the transition between catastrophic and stable shear banding occurs. When the diameter is smaller than the critical size of $\sim 0.93 \mu \mathrm{m}$, the strain energy raised by the flow stress is not high enough to allow for the shear band to fracture the pillar, thus, showing stable shear banding.

of a single shear band traversing the pillar. By equating Equations (1) and (2), $\Gamma$ is estimated to be $\sim 71 \mathrm{~J} \mathrm{~m}^{-2}$. Using the averaged flow stress of $\sim 4.2 \mathrm{GPa}$ as $\sigma_{\mathrm{c}}$, $\Gamma=71 \mathrm{~J} \mathrm{~m}^{-2}$ and $E=244.9 \mathrm{GPa}$ [62], the sample $d_{\mathrm{c}}$ was calculated from Equation (3) to be $\sim 0.93 \mu \mathrm{m}$ (Fig. 6), agreeing well with the experimental observations.

There are some similarities in the size-dependency of the deformation modes in the CrCoNi pillars identified in this study with that of metallic glass pillars [59]. However, different from metallic glass pillars, the steady shear banding behavior was observed in the CrCoNi pillars, which could be contributed to the hardening effect that slowed down the propagation of the shear bands. As shown in the inset in Fig. $2 \mathrm{f}$, the curve does not show a smooth stress response. The intermittent growth behavior of the single shear band suggests the coexistence of the softening and subsequent hardening events. In the early stages of shear banding, the dominant deformation mechanisms, such as detwinning, SF elimination, and HCP to FCC phase transformation, are considered to be strain softening processes, resulting in the stress drops to the valley of point 2 in Fig. 2 f. Strain softening is not always detrimental, since it is conducive to accommodate plasticity, and the high stress required to activate these softening mechanisms ensures the high yield strength. At the later stages, hardening mechanisms, such as the forma- tion of L-C locks and dislocation interactions, would kick in inside the shear band. The hardening mechanism is working to mitigate the softening effect, which could arrest the shear band propagation and increase the flow stress, e.g., from point 2 to 3 in Fig. $2 \mathrm{f}$, triggering more shear bands to bear further deformation. Therefore, a stable and prolonged plastic deformation process was attained in smaller pillars due to the development of numerous small and slowly propagated shear bands. However, in larger pillars, instantaneous occurrence of the shear event suppresses the strain hardening, which enables the softening effect to be dominant, inducing the sudden fracture of the pillar by the fast propagation of the shear bands. The transition from brittle to 'ductile' deformation (i.e., the increase of the deformability) with decreasing pillar diameters can thus be attributed to the decreased propagation ability and resulting increased amount of the shear bands.

Pillar geometry may influence the shear banding behavior, thus, the geometric factors, such as tapering, should be seriously considered when ascertaining the size effect on the deformation mode of pillars [63-65]. As shown in Fig. 7 , with comparable tapering angles of $\sim 4.3^{\circ}$, the larger pillar with a diameter of $1.25 \mu \mathrm{m}$ still exhibits a distinct catastrophic failure mode (Fig. 7b), while the steady shearing events can be apparently detected in the small pillar with a diameter of $0.4 \mu \mathrm{m}$ (Fig. $7 \mathrm{~d}$ ). Therefore, the brittle to 'ductile' transition observed in this study is governed by the intrinsic size effect, rather than an artifact.

\section{Phase transformation from HCP to FCC phase}

In the bulk CrCoNi-based HEAs and MEAs, previous studies have revealed that the phase transformation from FCC to HCP is, indeed, possible $[16,17,43,66]$. Theoretical investigations have proposed that the HCP phase is more thermodynamically stable than the FCC phase $[67,68]$ due to its low free energy and thus the formation of the HCP phase in the FCC phase is energetically favorable. However, experimental studies have revealed that the sophisticated generalized SFE curve of HEAs and MEAs due to the compositional complexity may kinetically hinder the phase transformation $[16,17,69]$, leading to only a limited quantity of the HCP phase that can transform from the FCC matrix. In striking contrast, the current study verified the unexpected reverse phase transformation from HCP to FCC in a dual-phased CrCoNi thin film at room temperature.

For all the pillars, a complete transformation from HCP to FCC is accomplished if a Shockley partial dislocation 

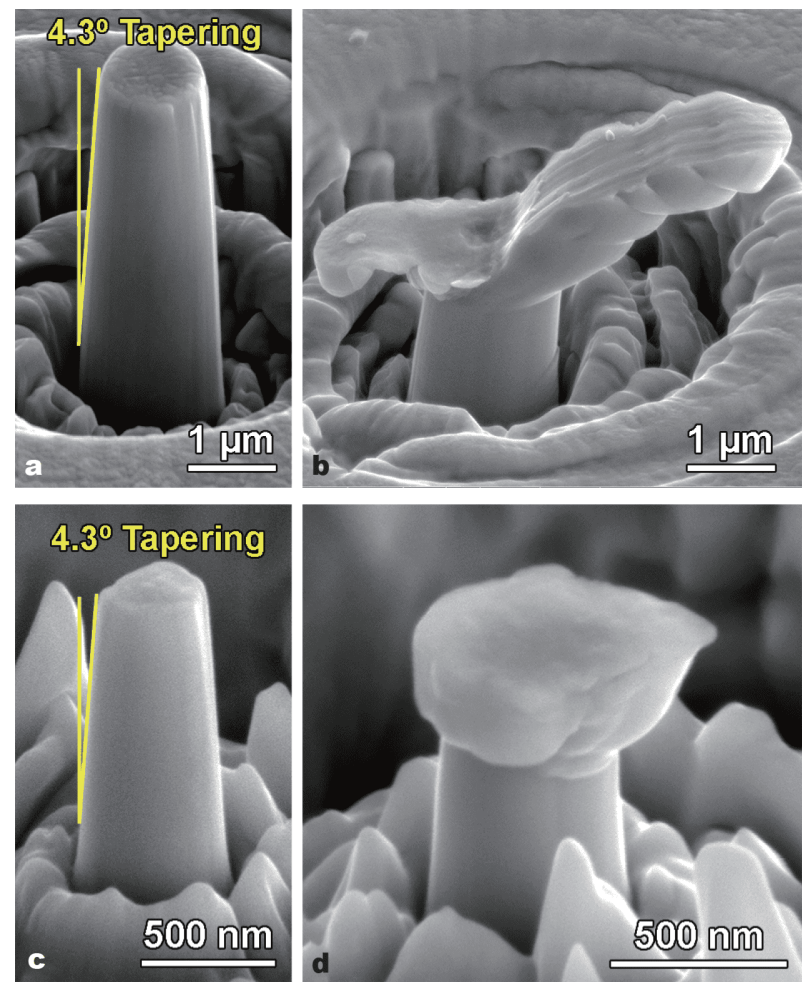

Figure 7 The size effect on the deformation mode remains even if the tests were conducted on larger pillars with the same degree of tapering as the smaller ones. SEM images of undeformed and post-compressed pillars with the same tapering angle of $4.3^{\circ}$, but different diameters of (a, b) $1.25 \mu \mathrm{m}$, and (c, d) $0.4 \mu \mathrm{m}$.

that creates a SF occurs on alternating close-packed planes in the HCP phase [70]. However, the driving force for the phase transformation in larger pillars and small (submicron) pillars could be different. In large pillars, an appreciable localized heating is estimated to occur if the stored elastic energy is catastrophically released in an avalanche configuration [71]. The catastrophic failure and the localized deformation would lead to an acceleration of strain rate, which might induce heat concentration and thermal softening in the shear band [72]. Unlike the vein- like patterns usually observed on the fracture plane of a metallic glass sample [73], the sheared surface of the large CrCoNi pillar (Fig. 2b) was marked with a striated pattern, which might be formed by softened metal surfaces rubbing against each other during shear deformation under an extremely high strain rate. This observation indicates that the significant rise of the temperature may result from fast, localized deformation within shear bands. Based on the theoretical calculations, for $\mathrm{CrCoNi}$, HCP is the stable structure at low temperature, while FCC becomes more stable when the temperature increases to above $~ 500 \mathrm{~K}$ [74]. Therefore, the HCP to FCC phase transformation observed in the catastrophically failed large pillars could be induced by the localized heating in the shear band.

For sub-micron pillars with steady deformation, the local temperature increase induced by dissipative heating during straining may not be high enough to drive the HCP to FCC phase transformation. The phase transformation in small pillar could be promoted by the high shear stress. At the early stage of deformation, multiple dislocations are nucleated at intersections between the planar defects and GBs/free surfaces [51,75], and then glide on the slip planes until they pile up against local obstacles (SFs and TBs). As the deformation proceeds, these piled-up dislocations start to react with the TBs and SFs when the shear stress is sufficiently large (Fig. $8 \mathrm{a}, \mathrm{b}$ ). Extensive dislocation-planar defects interactions enable the formation of shear bands with apparent crystal rotation (Fig. 8c) [51,75]. As shown in Fig. 8, the (0001) basal plane in the HCP phase is normal to the loading direction, thus there is no resolved shear stress available for driving dislocation motion on the basal plane. The crystallographic rotation caused by shear banding results in an increase in Schmidt factor and resolved shear stresses, thus facilitating the movement of partial dislocations on the basal plane of the HCP phase (Fig. 8d). Successive gliding of partials on every other basal plane of the HCP

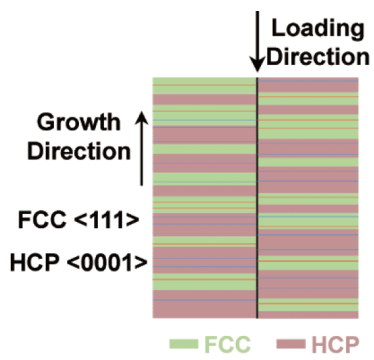

a

Original structure

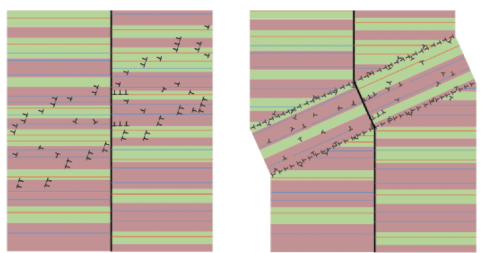

- TB - SF - Grain boundary

b

Dislocation pile-up

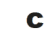

Geometric softening

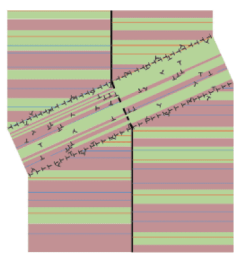

d

Dislocation

propagation

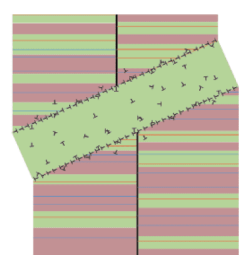

e

Phase

transformation

Figure 8 Schematic illustrations showing the structural evolution under shear banding in a nanostructured CrCoNi pillar. 
phase induces the phase transformation inside the shear band [70]. This rotation corresponds to geometric softening since it causes a lattice rotation into a geometrically softer orientation [76]. The large localized resolved shear stress caused by the lattice rotation inside the shear band is critical to the HCP to FCC phase transformation (Fig. 8e).

The HCP to FCC phase transformation in the nanostructured CrCoNi film might also be promoted by the high density of pre-existing SFs in the HCP phase, which act as nucleation sites for the formation of FCC segments at the beginning of deformation, overcoming the initial barrier seen from a planar-defect-free HCP phase transitioning to the FCC phase [77]. As reported by previous studies, the reverse martensitic transformation temperature can be lowered by the introduction of mechanical energy by deformation [78,79]. Noting that the original CrCoNi alloy has a high density of obstacles (GBs, TBs and SFs) for dislocation activities, thus, the mechanical energy cannot be easily released by means of dislocation propagation. A large amount of strain energy stored in the pillar might raise the free energy, providing the thermodynamic driving force for the HCP to FCC phase transformation, while further investigations are still needed to clarify the underlying mechanisms. All these results show that the HCP to FCC phase transformation in nanostructured $\mathrm{CrCoNi}$ is possible, and the HCP phase is mechanically unstable at room temperature. The HCP to FCC phase transformation leads to the formation of transparent HCP/FCC phase interface and softening. It is understood that strain softening would contribute to accommodate plasticity in the CrCoNi pillars.

\section{CONCLUSIONS}

The size effect on the deformation behavior of nanostructured, dual-phase $\mathrm{CrCoNi}$ alloy was examined by using in-situ compression testing, assisted by STEM analysis. The nanostructured CrCoNi MEA with hierarchal microstructures exhibits a ultrahigh sizeindependent yield strength of $\sim 4 \mathrm{GPa}$, which has its origins in the densely spaced planar defects including SFs, TBs and PBs. The compression tests of micro-pillars with different diameters showed that the pillars deformed mainly via shear banding due to the presence of nanoscale planar defects that limited the formation of mobile dislocations. As the pillar diameter decreased from 2 to $0.23 \mu \mathrm{m}$, the pillars showed transitions from highly localized shear banding, through steady shear banding, and to homogeneous deformation that promoted significant plasticity. An unusual phase transformation from the HCP to FCC phase was observed in the heavily deformed regions mediated by Shockley partial dislocations. The high density SFs presented in the CrCoNi alloy are believed to facilitate the HCP to FCC phase transformation.

Received 28 March 2020; accepted 29 April 2020;

published online 29 July 2020

1 Yeh JW, Chen SK, Lin SJ, et al. Nanostructured high-entropy alloys with multiple principal elements: novel alloy design concepts and outcomes. Adv Eng Mater, 2004, 6: 299-303

2 Cantor B, Chang ITH, Knight P, et al. Microstructural development in equiatomic multicomponent alloys. Mater Sci Eng-A, 2004, 375-377: 213-218

3 Zhang Y, Zuo TT, Tang Z, et al. Microstructures and properties of high-entropy alloys. Prog Mater Sci, 2014, 61: 1-93

4 Zhang W, Liaw PK, Zhang Y. Science and technology in highentropy alloys. Sci China Mater, 2018, 61: 2-22

5 Gludovatz B, Hohenwarter A, Catoor D, et al. A fracture-resistant high-entropy alloy for cryogenic applications. Science, 2014, 345: $1153-1158$

6 Lin $\mathrm{Q}$, An $\mathrm{X}$, Liu $\mathrm{H}$, et al. In-situ high-resolution transmission electron microscopy investigation of grain boundary dislocation activities in a nanocrystalline $\mathrm{CrMnFeCoNi}$ high-entropy alloy. J Alloys Compd, 2017, 709: 802-807

7 Otto F, Yang Y, Bei H, et al. Relative effects of enthalpy and entropy on the phase stability of equiatomic high-entropy alloys. Acta Mater, 2013, 61: 2628-2638

8 Ma D, Grabowski B, Körmann F, et al. Ab initio thermodynamics of the CoCrFeMnNi high entropy alloy: Importance of entropy contributions beyond the configurational one. Acta Mater, 2015, 100: 90-97

9 Yang X, Zhang Y. Prediction of high-entropy stabilized solidsolution in multi-component alloys. Mater Chem Phys, 2012, 132: 233-238

10 Li Z, Pradeep KG, Deng Y, et al. Metastable high-entropy dualphase alloys overcome the strength-ductility trade-off. Nature, 2016, 534: 227-230

11 Lu W, Liebscher CH, Dehm G, et al. Bidirectional transformation enables hierarchical nanolaminate dual-phase high-entropy alloys. Adv Mater, 2018, 30: 1804727

12 An XH, Wu SD, Wang ZG, et al. Significance of stacking fault energy in bulk nanostructured materials: insights from $\mathrm{Cu}$ and its binary alloys as model systems. Prog Mater Sci, 2019, 101: 1-45

13 Liu J, Guo X, Lin Q, et al. Excellent ductility and serration feature of metastable $\mathrm{CoCrFeNi}$ high-entropy alloy at extremely low temperatures. Sci China Mater, 2019, 62: 853-863

14 Gludovatz B, Hohenwarter A, Thurston KVS, et al. Exceptional damage-tolerance of a medium-entropy alloy $\mathrm{CrCoNi}$ at cryogenic temperatures. Nat Commun, 2016, 7: 10602

15 Niu C, LaRosa CR, Miao J, et al. Magnetically-driven phase transformation strengthening in high entropy alloys. Nat Commun, 2018, 9: 1363

16 Ma Y, Yuan F, Yang M, et al. Dynamic shear deformation of a CrCoNi medium-entropy alloy with heterogeneous grain structures. Acta Mater, 2018, 148: 407-418

17 Miao J, Slone CE, Smith TM, et al. The evolution of the deformation substructure in a $\mathrm{Ni}-\mathrm{Co}-\mathrm{Cr}$ equiatomic solid solution 
alloy. Acta Mater, 2017, 132: 35-48

18 Schuh B, Mendez-Martin F, Völker B, et al. Mechanical properties, microstructure and thermal stability of a nanocrystalline $\mathrm{CoCr}$ FeMnNi high-entropy alloy after severe plastic deformation. Acta Mater, 2015, 96: 258-268

19 Slone CE, Miao J, George EP, et al. Achieving ultra-high strength and ductility in equiatomic $\mathrm{CrCoNi}$ with partially recrystallized microstructures. Acta Mater, 2019, 165: 496-507

20 Sun SJ, Tian YZ, An XH, et al. Ultrahigh cryogenic strength and exceptional ductility in ultrafine-grained CoCrFeMnNi highentropy alloy with fully recrystallized structure. Mater Today Nano, 2018, 4: 46-53

21 He JY, Wang H, Huang HL, et al. A precipitation-hardened highentropy alloy with outstanding tensile properties. Acta Mater, 2016, 102: 187-196

22 Guo L, Gu J, Gong X, et al. CALPHAD aided design of high entropy alloy to achieve high strength via precipitate strengthening. Sci China Mater, 2020, 63: 288-299

23 Yang T, Zhao YL, Tong Y, et al. Multicomponent intermetallic nanoparticles and superb mechanical behaviors of complex alloys. Science, 2018, 362: 933-937

24 Seol JB, Bae JW, Li Z, et al. Boron doped ultrastrong and ductile high-entropy alloys. Acta Mater, 2018, 151: 366-376

25 Li Z, Tasan CC, Springer H, et al. Interstitial atoms enable joint twinning and transformation induced plasticity in strong and ductile high-entropy alloys. Sci Rep, 2017, 7: 40704

26 Song $\mathrm{M}$, Zhou R, Gu J, et al. Nitrogen induced heterogeneous structures overcome strength-ductility trade-off in an additively manufactured high-entropy alloy. Appl Mater Today, 2020, 18: 100498

27 Wang Z, Gu J, An D, et al. Characterization of the microstructure and deformation substructure evolution in a hierarchal highentropy alloy by correlative EBSD and ECCI. Intermetallics, 2020, 121: 106788

28 Lei Z, Liu X, Wu Y, et al. Enhanced strength and ductility in a high-entropy alloy via ordered oxygen complexes. Nature, 2018, 563: 546-550

29 Chen Y, Zhou Z, Munroe P, et al. Hierarchical nanostructure of CrCoNi film underlying its remarkable mechanical strength. Appl Phys Lett, 2018, 113: 081905

30 Tsianikas SJ, Chen Y, Xie Z. Deciphering deformation mechanisms of hierarchical dual-phase CrCoNi coatings. J Mater Sci Tech, 2020, 39: 7-13

31 Uchic MD, Dimiduk DM, Florando JN, et al. Sample dimensions influence strength and crystal plasticity. Science, 2004, 305: 986989

32 Greer JR, Nix WD. Nanoscale gold pillars strengthened through dislocation starvation. Phys Rev B, 2006, 73: 245410

33 Shan ZW, Mishra RK, Syed Asif SA, et al. Mechanical annealing and source-limited deformation in submicrometre-diameter $\mathrm{Ni}$ crystals. Nat Mater, 2008, 7: 115-119

34 Wang ZJ, Li QJ, Shan ZW, et al. Sample size effects on the large strain bursts in submicron aluminum pillars. Appl Phys Lett, 2012, 100: 071906

35 Nix WD, Greer JR, Feng G, et al. Deformation at the nanometer and micrometer length scales: effects of strain gradients and dislocation starvation. Thin Solid Films, 2007, 515: 3152-3157

36 Hütsch J, Lilleodden ET. The influence of focused-ion beam preparation technique on microcompression investigations: Lathe $v s$. annular milling. Scripta Mater, 2014, 77: 49-51
37 Guo W, Jägle E, Yao J, et al. Intrinsic and extrinsic size effects in the deformation of amorphous $\mathrm{CuZr}$ /nanocrystalline $\mathrm{Cu}$ nanolaminates. Acta Mater, 2014, 80: 94-106

38 Chen CQ, Pei YT, De Hosson JTM. Effects of size on the mechanical response of metallic glasses investigated through in situ TEM bending and compression experiments. Acta Mater, 2010, 58: 189-200

39 Giannuzzi LA, Stevie FA. A review of focused ion beam milling techniques for TEM specimen preparation. Micron, 1999, 30: 197204

40 Greer JR, De Hosson JTM. Plasticity in small-sized metallic systems: intrinsic versus extrinsic size effect. Prog Mater Sci, 2011, 56: 654-724

41 Hull D, Bacon DJ. Introduction to Dislocations. Oxford: Elsevier, 2011

42 Liu SF, Wu Y, Wang HT, et al. Stacking fault energy of facecentered-cubic high entropy alloys. Intermetallics, 2018, 93: 269273

43 Lin Q, Liu J, An X, et al. Cryogenic-deformation-induced phase transformation in an FeCoCrNi high-entropy alloy. Mater Res Lett, 2018, 6: 236-243

44 Schuh B, Völker B, Todt J, et al. Influence of annealing on microstructure and mechanical properties of a nanocrystalline CrCoNi medium-entropy alloy. Materials, 2018, 11: 662

45 Zhu ZG, Nguyen QB, Ng FL, et al. Hierarchical microstructure and strengthening mechanisms of a CoCrFeNiMn high entropy alloy additively manufactured by selective laser melting. Scripta Mater, 2018, 154: 20-24

46 Lu L, Chen X, Huang X, et al. Revealing the maximum strength in nanotwinned copper. Science, 2009, 323: 607-610

47 Lu K, Lu L, Suresh S. Strengthening materials by engineering coherent internal boundaries at the nanoscale. Science, 2009, 324 349-352

48 Chen B, Wang J, Gao Q, et al. Strengthening brittle semiconductor nanowires through stacking faults: insights from in situ mechanical testing. Nano Lett, 2013, 13: 4369-4373

49 Chen Y, Burgess T, An X, et al. Effect of a high density of stacking faults on the Young's modulus of GaAs nanowires. Nano Lett, 2016, 16: 1911-1916

50 Li Z, Tasan CC, Pradeep KG, et al. A trip-assisted dual-phase highentropy alloy: grain size and phase fraction effects on deformation behavior. Acta Mater, 2017, 131: 323-335

51 Wang J, Sansoz F, Huang J, et al. Near-ideal theoretical strength in gold nanowires containing angstrom scale twins. Nat Commun, 2013, 4: 1742

52 Wang L, Lu Y, Kong D, et al. Dynamic and atomic-scale understanding of the twin thickness effect on dislocation nucleation and propagation activities by in situ bending of $\mathrm{Ni}$ nanowires. Acta Mater, 2015, 90: 194-203

53 Jang D, Greer JR. Size-induced weakening and grain boundaryassisted deformation in $60 \mathrm{~nm}$ grained Ni nanopillars. Scripta Mater, 2011, 64: 77-80

54 Chen CQ, Pei YT, Kuzmin O, et al. Intrinsic size effects in the mechanical response of taper-free nanopillars of metallic glass. Phys Rev B, 2011, 83: 180201

55 Shan ZW, Li J, Cheng YQ, et al. Plastic flow and failure resistance of metallic glass: insight from in situ compression of nanopillars. Phys Rev B, 2008, 77: 155419

56 Kuzmin OV, Pei YT, Chen CQ, et al. Intrinsic and extrinsic size effects in the deformation of metallic glass nanopillars. Acta Mater, 
2012, 60: 889-898

57 Khalajhedayati A, Rupert TJ. Disruption of thermally-stable nanoscale grain structures by strain localization. Sci Rep, 2015, 5: 10663

58 Rice JR. The localization of plastic deformation. In: Proceedings of the 14th International Congress of Theoretical and Applied Mechanics. Delft, 1976. 1-14

59 Volkert CA, Donohue A, Spaepen F. Effect of sample size on deformation in amorphous metals. J Appl Phys, 2008, 103: 083539

60 Jang D, Greer JR. Transition from a strong-yet-brittle to a stronger-and-ductile state by size reduction of metallic glasses. Nat Mater, 2010, 9: 215-219

61 Gao H, Ji B, Jager IL, et al. Materials become insensitive to flaws at nanoscale: lessons from nature. Proc Natl Acad Sci USA, 2003, 100: 5597-5600

62 Laplanche G, Gadaud P, Bärsch C, et al. Elastic moduli and thermal expansion coefficients of medium-entropy subsystems of the CrMnFeCoNi high-entropy alloy. J Alloys Compd, 2018, 746: 244255

63 Wu XL, Guo YZ, Wei Q, et al. Prevalence of shear banding in compression of $\mathrm{Zr}_{41} \mathrm{Ti}_{14} \mathrm{Cu}_{12.5} \mathrm{Ni}_{10} \mathrm{Be}_{22.5}$ pillars as small as $150 \mathrm{~nm}$ in diameter. Acta Mater, 2009, 57: 3562-3571

64 Schuster BE, Wei Q, Hufnagel TC, et al. Size-independent strength and deformation mode in compression of a Pd-based metallic glass. Acta Mater, 2008, 56: 5091-5100

65 Chen Y, An X, Liao X, et al. Effects of loading misalignment and tapering angle on the measured mechanical properties of nanowires. Nanotechnology, 2015, 26: 435704

66 Laplanche G, Kostka A, Reinhart C, et al. Reasons for the superior mechanical properties of medium-entropy $\mathrm{CrCoNi}$ compared to high-entropy CrMnFeCoNi. Acta Mater, 2017, 128: 292-303

67 Zhang ZJ, Mao MM, Wang J, et al. Nanoscale origins of the damage tolerance of the high-entropy alloy CrMnFeCoNi. Nat Commun, 2015, 6: 10143

68 Huang S, Huang H, Li W, et al. Twinning in metastable highentropy alloys. Nat Commun, 2018, 9: 2381

69 Zhang FX, Zhao S, Jin K, et al. Pressure-induced FCC to HCP phase transition in Ni-based high entropy solid solution alloys. Appl Phys Lett, 2017, 110: 011902

70 Zhao H, Song M, Ni S, et al. Atomic-scale understanding of stressinduced phase transformation in cold-rolled Hf. Acta Mater, 2017, 131: 271-279

71 Armstrong RW, Coffey CS, Elban WL. Adiabatic heating at a dislocation pile-up avalanche. Acta Metall, 1982, 30: 2111-2116

72 Wei Q, Jia D, Ramesh KT, et al. Evolution and microstructure of shear bands in nanostructured Fe. Appl Phys Lett, 2002, 81: 12401242

73 Zhang ZF, Eckert J, Schultz L. Difference in compressive and tensile fracture mechanisms of $\mathrm{Zr}_{59} \mathrm{Cu}_{20} \mathrm{Al}_{10} \mathrm{Ni}_{8} \mathrm{Ti}_{3}$ bulk metallic glass. Acta Mater, 2003, 51: 1167-1179

74 Zhang Z, Sheng H, Wang Z, et al. Dislocation mechanisms and 3D twin architectures generate exceptional strength-ductility-toughness combination in CrCoNi medium-entropy alloy. Nat Commun, 2017, 8: 14390

75 Jang D, Li X, Gao H, et al. Deformation mechanisms in nanotwinned metal nanopillars. Nat Nanotech, 2012, 7: 594-601

76 Asaro RJ. Geometrical effects in the inhomogeneous deformation of ductile single crystals. Acta Metall, 1979, 27: 445-453

77 Fujita H, Ueda S. Stacking faults and f.c.c. $(\gamma) \rightarrow$ h.c.p. $(\varepsilon)$ transformation in 188-type stainless steel. Acta Metall, 1972, 20: 759-
767

78 Huang JY, Wu YK, Ye HQ. Allotropic transformation of cobalt induced by ball milling. Acta Mater, 1996, 44: 1201-1209

79 Liu Y, Yang H, Tan G, et al. Stress-induced FCC $\leftrightarrow$ HCP martensitic transformation in CoNi. J Alloys Compd, 2004, 368: 157163

Acknowledgements This work was supported by the Australian Research Council Discovery Projects Grant, and partly supported by the Fundamental Research Funds for the Central Universities (SWU118105). An X acknowledges the financial support from Australia Research Council (DE170100053) and the Robinson Fellowship Scheme of the University of Sydney (G200726). The authors acknowledge the facilities and the scientific and technical assistance of the Australian Microscopy and Microanalysis Research Facility (ammrf.org.au) node at the University of Adelaide: Adelaide Microscopy. In particular, the authors thank Dr Animesh Basak and Dr Ashley Slattery of Adelaide Microscopy for their support and expertise.

Author contributions Chen $\mathrm{Y}, \mathrm{Xie} \mathrm{Z}$, An X and Zhang S conceived the project. Chen $\mathrm{Y}$ conducted the FIB, microcompression and TEM experiments. Zhou Z fabricated the samples. Chen Y, An X, Liao X and Xie $\mathrm{Z}$ interpreted the results and wrote the manuscript. All authors contributed to the discussion of the results, and comments on the manuscript.

Conflict of interest The authors declare that they have no conflict of interest.

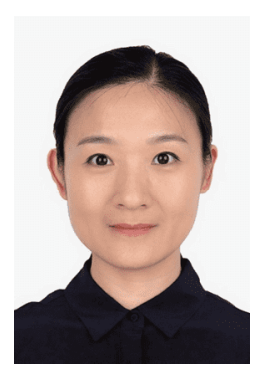

Yujie Chen obtained her BEng degree (first-class honors) in 2011 and $\mathrm{PhD}$ degree in materials science in 2016 from The University of Sydney. Upon completion of her $\mathrm{PhD}$, she was employed as a postdoc in the School of Mechanical Engineering in the University of Adelaide in Australia. She is currently a research fellow in the Southwest University in China. Her current research involves microstructure optimization and mechanical properties enhancement of alloys, and calcified tissues.

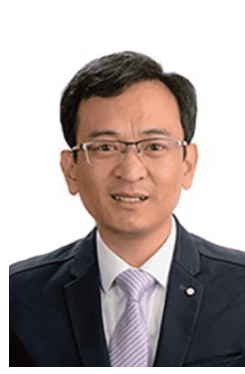

Xianghai An received his $\mathrm{PhD}$ degree from the Institute of Metal Research, Chinese Academy of Sciences in 2012. After receiving his $\mathrm{PhD}$ degree, he commenced to work as a research fellow at The University of Sydney. He is currently a Lecture/Robinson Fellow at The University of Sydney. His research mainly focuses on materials design, mechanical behavior, and structureproperty relationship of advanced metallic materials, nanomechanics and nanoplasticity, metallic additive manufacturing and advanced 


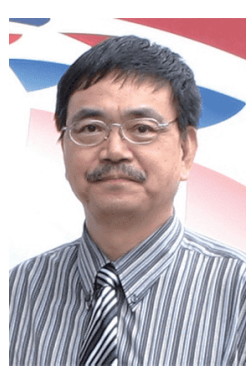

Sam Zhang received his $\mathrm{PhD}$ degree (1991) in ceramic materials at the University of WisconsinMadison, USA. He joined Nanyang Technological University as an associate professor and was promoted to full professor in 2006. He is currently a professor and head of the Center for Advanced Thin Films and Devices in the Southwest University in China. He is also Fellow of the Institute of Materials, Minerals and Mining, Fellow of the Royal Society of Chemistry and Fellow of the Thin Films Society. His research interests include preparation and characterization of hard yet tough ceramic nanocomposite coatings, and functional thin films.

\section{双相纳米结构 $\mathrm{CrCoNi}$ 中熵合金变形行为的尺寸 效应}

陈玉洁 ${ }^{1,2,3}$, 安祥海 ${ }^{3^{*}}$, 周志烽 ${ }^{4}$, Paul Munroe ${ }^{5}$, 张善勇 ${ }^{{ }^{*}}$, 廖晓舟 ${ }^{3}$, 谢宗翰 ${ }^{2,6}$

摘要 本文结合原位扫描电子显微镜微柱压缩与透射电子显微镜 技术，研究了具有双相多级纳米结构的 $\mathrm{CrCoNi}$ 中商合金变形行为 的尺寸效应. 研究表明, 该合金的屈服强度高达 4 GPa, 这主要归 因于其多级微观结构特征, 包括柱状纳米尺寸晶粒、织构、高密 度的层错、孪晶界、晶界和相界. 在变形过程中, 该合金的屈服强 度基本与微米尺度样品的尺寸无关，但其变形行为却强烈依赖于 样品大小. 具体来说, 随着微柱直径减小, 材料主要的变形模式从 突发的局部剪切带变为具有明显塑性的均匀变形. 这种转变是由 剪切带穿过微柱所需的临界应力与样品尺寸紧密相关所决定的, 剪切诱导的软化和随后的硬化机制之间的竞争也起了重要作用. 此外, 变形引起了六方密排结构到面心立方结构的相变, 该相变导 致的应变软化对材料变形中的塑性有重要贡献. 这些发现揭示了 样品尺寸对可用于微纳机电系统的纳米结构金属材料的力学行为 有着重要影响. 